
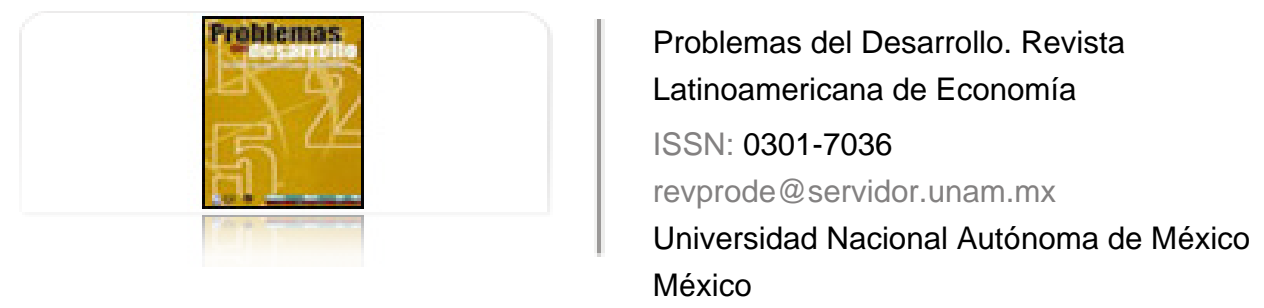

Ramírez Cedillo, Eduardo

La política fiscal desde una perspectiva de crecimiento endógeno, equilibrio presupuestal y fluctuaciones de corto plazo

Problemas del Desarrollo. Revista Latinoamericana de Economía, vol. 39, núm. 152, enero-marzo, 2008, pp. 113-137

Universidad Nacional Autónoma de México

Distrito Federal, México

Disponible en: http://www.redalyc.org/articulo.oa?id=11820668007

Cómo citar el artículo

- Número completo

- Más información del artículo

Página de la revista en redalyc.org

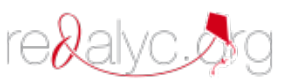

Sistema de Información Científica

Red de Revistas Científicas de América Latina, el Caribe, España y Portugal Proyecto académico sin fines de lucro, desarrollado bajo la iniciativa de acceso abierto 


\title{
LA POLIITICA FISCAL DESDE UNA PERSPECTIVA DE CRECIMIENTO ENDÓGENO, EQUILIBRIO PRESUPUESTAL Y FLUCTUACIONES DE CORTO PLAZO
}

\author{
Eduardo Ramírez Cedillo*
}

Fecha de recepción: 31 de mayo de 2007. Fecha de aceptación: 20 de diciembre de 2007.

\section{Resumen}

La relación de la política fiscal $(P F)$ y el crecimiento económico se ha abordado mediante el desarrollo de un cuerpo teórico-práctico denominado "modelos de crecimiento endógeno (MCE)", que asumen que la PF puede incidir en el crecimiento, siempre y cuando los instrumentos de aplicación de gasto y la captación de los recursos observen determinadas características. Por el lado del gasto se considera que existen algunos tipos de erogaciones que son productivas y otras que no lo son, mientras que por el lado de los ingresos se observa que se tienen ingresos distorsionadores y no distorsionadores. A pesar de que el presente documento coincide con algunos postulados de los $M C E$, la divergencia se sitúa en la poca atención que se le da a la posibilidad de que la PF pueda incurrir en déficit, al teorizar principalmente sobre un contexto de finanzas públicas en equilibrio.

Palabras clave: política fiscal, modelos de crecimiento endógeno, gasto de gobierno, ingresos públicos, déficit presupuestal.

* Profesor en la Facultad de Contaduría y Administración y la Facultad de Economía en la UNAM. Correo electrónico: ramceed@ prodigy.net.mx. 


\section{Summary}

The relationship between fiscal policy $(F P)$ and economic growth has been dealt with using a theoretico-practical corpus known as "models of endogenous growth (MEG), which assumes that FP can affect growth, as long as the instruments for applying spending and the inflow of resources carry certain characteristics. On the spending side, it is thought that some kinds of outlay are productive and others are not, while on the income side it is assumed that incomes can either be distortional or non-distortional. Although the present document agrees with some of the views postulated by MEG, it diverges from it due to its lack of attention to the possibility that FP can result in a deficit, looking basically at a context of public finance in equilibrium.

Key words: fiscal policy, models of endogenous growth, government spending, public income, budget deficit.

\section{Résumé}

Le rapport entre la politique fiscale $(P F)$ et la croissance économique a été abordé au travers du développement d'un corpus théorico-pratique dénommé "modèles de croissance endogène (MCE)", selon lesquels la PF peut avoir une incidence sur la croissance à condition que les instruments d'application de dépense et la captation des ressources présentent certaines caractéristiques. En ce qui concerne les dépenses, il est considéré qu'il en existe de productives et d'autres qui ne le sont pas, tandis que pour les recettes, il est pris le parti que certaines ont un effet de distorsion et d'autres non. Bien que ce travail coüncide avec certains postulats des MCE, il en diverge par l'attention qu'il porte à la possibilité que la PF participe au déficit si elle se fonde principalement sur un contexte de finances publiques équilibrées.

Mots clés: politique fiscale, modèles de croissance endogène, dépenses du gouvernement, recettes publiques, déficit budgétaire.

\section{Resumo}

A relação entre a política fiscal $(P F)$ e o crescimento econômico tem sido abordada mediante o desenvolvimento de um corpo teórico-práctico denominado "modelos de crescimento endógeno (MCE)", que assumem que a PF pode incidir no crescimento, sempre que os instrumentos de aplicação de gasto e a captação de recursos observem determinadas características. Pelo lado do gasto considera-se que existem alguns tipos de contribuições que são produtivas e outras que não o são, enquanto que pelo lado dos insumos assume-se que há insumos distorcionadores y não-distorcionadores. Apesar de que o presente documento coincide com alguns postulados dos MCE, a divergência situa-se na pouca atenção que se dá à possibilidade de que a PF possa incorrer em déficit, ao teorizar principalmente sobre um contexto de finanças públicas em equilíbrio.

Palavras-chave: política fiscal, modelos de crescimento endógeno, gasto de governo, ingressos públicos, déficit orçamentário.

\section{Desarrrollo}




\section{Introducción}

$\mathrm{n}$ años recientes ha surgido una vasta literatura respecto a la relación entre la
política fiscal y el crecimiento económico. Muchos de estos planteamientos
se han cimentado en la teoría del crecimiento endógeno que, a diferencia de los modelos neoclásicos, le asigna un papel relevante a la política fiscal (PF). Para los modelos de crecimiento endógeno (MCE) la inversión en capital humano y la investigación y desarrollo resultan ser las piezas clave en el crecimiento económico, pues generan nuevos productos o ideas bajo las cuales se da el progreso tecnológico que dinamiza la economía y alienta el crecimiento (Romer, 1990; Barro, 1991). Desde este planteamiento se ha incorporado la participación del sector gubernamental, al aducir que la tasa de crecimiento y ahorro se incrementan cuando lo hace la relación gasto de gobierno-PIB, pero esto sólo funciona hasta un límite y después declina (Barro, 1990; Barro y Sala-i-Martín, 1992). Por otra parte, diversas investigaciones se han centrado en los efectos de los impuestos sobre el crecimiento económico, donde se destaca que en algunos casos los impuestos altos no son necesariamente malos (Engen y Skinner, 1996), o bien que su efecto sobre el crecimiento tiende a ser débil (Myles, 2000; Easterly y Rebelo, 1994). Otros estudios han abordado tanto los gastos como los ingresos del gobierno, es decir, la $\mathrm{PF}$ en su conjunto. Los resultados han sido distintos y se puede encontrar evidencia tanto a favor como en contra. La evidencia en contra se localiza en escritos como Engen y Skinner (1992), mientras que los argumentos a favor se ubican en Easterly y Rebelo (1994) y Gemmell (2001). Otro punto de discusión con base en la relación PF-crecimiento económico consiste en la duración de los efectos de la PF sobre el crecimiento, es decir, si es de corto o largo plazo. Zagler y Durnecker (2003) comentan que los efectos de la PF en el corto plazo son reconocidos porque pueden mitigar las fluctuaciones de crecimiento y desempleo, ${ }^{1}$ no obstante en el largo plazo se piensa que pueden llegar a distorsionar la economía al crear desequilibrios, por lo que es importante estudiar con más detalle el largo plazo. En este sentido, Engen y Skinner (1992) mencionan que bajo la argumentación neoclásica la imposición de contribuciones y el gasto de gobierno no tienen impacto en el largo plazo y su

1 El problema macroeconómico de corto plazo es la inadecuada demanda agregada de bienes y servicios que pueden ser producidos por las empresas privadas y ello ocasiona una menor tasa de ocupación de la capacidad instalada. Desde esta consideración Gale y Orszag (2003) dicen que la política que genera déficit presupuestal de carácter temporal puede estimular la demanda agregada y el mejor desarrollo económico de corto plazo. 
efecto se limita sólo a los niveles del producto. ${ }^{2}$ No obstante, Gemmell (2001), aludiendo la teoría del crecimiento endógeno, expresa que la PF puede tener efectos de largo plazo.

En los MCE se consideran como detonadores del crecimiento económico algunas acciones del gobierno. ${ }^{3}$ Desde esta óptica se piensa que el crecimiento dependerá de los instrumentos de política económica que se utilicen, por tanto es necesario distinguir entre gastos productivos e improductivos, e impuestos distorsionadores y no distorsionadores (Gemmell, 2001).

Cuando los MCE asumen un equilibrio presupuestal limitan la posibilidad de los países para lograr incidir en el elemento de corto plazo del crecimiento económico, pues en cierto sentido el gasto de gobierno y la recaudación tributaria podrían tener un comportamiento pro-cíclico que acentuaría la volatilidad de la economía y podrían jugar en contra del crecimiento económico de largo plazo, tal como lo ha comentado Martner (1998) y como se desprende de Ramey y Ramey (1994).

Desde la perspectiva de los MCE y el avance en la diferenciación del gasto gubernamental para alentar el crecimiento se pueden llegar a resultados importantes, pero en materia de tributos se genera una limitante teórica al asumirlos tan sólo como fuentes de financiamiento, dejando de lado la consideración de que pueden y deben cumplir con papeles económicos más amplios, como son descritos por Lerner (1947), Wray (1998), Bell (1998) y Ramírez (2007).

El objetivo de este documento se ubica en dos vertientes principales: por una parte la descripción teórica sobre el uso de los instrumentos de PF (gasto-ingreso) que es consistente con el planteamiento de los MCE; por la otra se intenta teorizar cómo los efectos de la PF de corto plazo denotados por el ciclo económico pueden poner en evidencia las perspectivas de largo plazo, sobre todo en aquellos casos en los que se parte de finanzas públicas sanas, capacidad excedente y se limita el papel de los impuestos a ser tan sólo instrumentos de financiamiento del gasto gubernamental. Por último, se presentan las conclusiones.

2 Easterly y Rebelo (1994) lo argumentan de la siguiente forma: "en los modelos neoclásicos el crecimiento es conducido por factores exógenos, la PF sólo puede afectar la tasa de crecimiento durante la transición al estado de equilibrio. La sabiduría convencional basada en modelos neoclásicos ha sido que la diferencia entre sistema impositivo, la deuda y la política de gasto pueden ser importantes en la determinación de los niveles de producto, pero es improbable que tenga un efecto importante en la tasa de crecimiento".

3 Easterly y Rebelo, ibid., comentan que en los MCE, la PF puede ser uno de los determinantes importantes para observar diferencias en las experiencias de crecimiento. Los MCE tienden a transformar los efectos temporales de la PF sobre el crecimiento en efectos permanentes. 


\section{Descripción de los instrumentos}

Los MCE han tratado de establecer una égida en la conjugación que de sus instrumentos deben hacer la PF para lograr el crecimiento económico, tomando como principales conceptos el gasto gubernamental y la recaudación de impuestos. Para estas dos acciones principales se ha matizado un esquema de funcionamiento que se detalla a continuación.

\section{Gasto de gobierno}

En la literatura hay coincidencia entre diversos autores sobre el suministro de bienes públicos (non-market) como una actividad legítima en la que debe participar el gobierno, pero de manera adicional, bajo el planteamiento de los MCE (Romer 1990, Barro 1990). Se piensa que también debe propiciar la formación de capital humano, mediante la participación del Estado en el suministro de educación y salud. Además la inversión en capital físico jugará un papel importante en las expectativas de crecimiento de una economía.

Lo anterior no es nuevo, aun cuando podría parecerlo, pues derivado de la tradición keynesiana se ha realizado una mala interpretación de sus ideas, al pensar que su planteamiento se refería al uso desenfrenado de recursos por parte del Estado para salir de periodos recesivos o bien propiciar mayores niveles de crecimiento. Camara Neto y Venergo (2004), entre otros, son claros al mencionar que en la perspectiva de Keynes era necesario realizar una distinción entre el gasto corriente y el gasto de capital para asegurar que la provisión de servicios del gobierno no fueran subsidiados con recursos impropios, previendo con ello el riesgo moral, mientras el segundo bien podría ser usado de forma contra-cíclica.

La clasificación del gasto

Para examinar los posibles efectos del gasto de gobierno en el crecimiento económico, es conveniente clasificarlo en dos grandes rubros que son el gasto corriente y de capital, como propone Keynes. El gasto corriente puede llegar a entorpecer el crecimiento económico, mientras que el gasto de capital lo propicia (Zagler y Durnecker, 2003). La diferencia entre los dos tipos de gasto es la consideración de que el consumo implica una erogación inmediata, mientras que los gastos de capital proporcionan un retorno real con el tiempo (Camara Neto y Venergo, 2004); sin embargo, esta clasificación es insuficiente en virtud de sus complicaciones para determinar qué se considera como gasto corriente y qué se asume como gasto de capital. 
$\mathrm{Al}$ abundar en los efectos explicativos de cierto tipo de gasto corriente en el crecimiento de la economía, se argumenta que hay dos canales por los cuales el gobierno puede incrementar la productividad: la acumulación de conocimientos y los cuidados a la salud (Zagler y Durnecker, 2003).

Con la intención de solventar el inconveniente que presenta la clasificación corriente-capital, se propone dividir el gasto público en productivo e improductivo, donde el primero tiene una fuerte capacidad para lograr el crecimiento económico, mientras que el segundo se refiere puramente al consumo (Devajaran et al., 1996); la ventaja de esta propuesta consiste en la posibilidad de valorar el gasto del gobierno en su justa dimensión, teniendo en cuenta que en algunos casos el gasto productivo (educación) puede observar largos periodos para ofrecer rentabilidad. Gemmell (2001) apunta que en el caso de los países con bajo nivel de ingreso (Low Income Countries: LIC) la importancia radica en que se ponga atención e identifique qué clase de gasto público es considerado como "productivo", pues en países que se recuperan de choques derivados de guerras o la reconstrucción de su capital humano puede ser que mucho de su gasto esté más relacionado con el gasto corriente que con el capital. Por tanto, para los LIC, una distinción entre la relación capital-corriente no debería ser asociada con una distinción entre la relación productividad-improductividad.

Al hablar un poco más en la distinción consumo-capital, es importante mencionar que el gasto asociado a programas sociales normalmente es tomado como gasto de consumo, que no aportan ventajas a la producción presente o futura y tiene la peculiaridad de ser puramente asistencial. No obstante, Alesina y Perotti (1996) encuentran evidencia empírica al respecto de cómo la desigualdad económica genera malestar social e inestabilidad política, lo que puede deprimir la inversión privada; por tanto, el gasto social lejos de desalentar el crecimiento puede ser su base en los LIC.

El gasto que el gobierno realiza en materia de infraestructura (inversión de capital) tiene un potencial diferente al que se lleva a cabo en materia de educación o salud; Aschauer (1989a) indica que el gasto gubernamental en este rubro — construcción de calles, carreteras, medios masivos de transporte, alcantarillado, sistemas de provisión de agua, etcétera - tiene un alto poder de explicación para la productividad del conjunto de la economía. Por su parte Easterly y Rebelo (1994) coinciden en precisar que el gasto de inversión del gobierno en comunicaciones y transportes está consistentemente correlacionado con el crecimiento económico de un país. Tridimas (1992) adiciona que la inversión del sector público, dedicada a proporcionar infraestructura a la economía, incrementa la acumulación de capital y aumenta el ingreso permanente. 
La participación del gobierno en la economía dentro de un modelo de crecimiento endógeno puede ser representada de la siguiente forma para un sector competitivo que produce bienes finales: $Y_{t}$, que son producidos usando trabajo $L_{t}$, insumos intermedios especializados $\mathrm{X}_{\mathrm{t}}$ y la provisión de bienes públicos $\mathrm{G}_{\mathrm{t}}$.

$$
Y_{t}=X_{t}^{\alpha} G_{t}^{\beta} L_{t}^{1-\alpha}
$$

Un incremento en el gasto de gobierno aumenta directamente la productividad marginal de la contribución de los factores privados que alientan su acumulación y, por tanto, puede inducir el crecimiento del producto.

Se tiene entonces evidencia que habla de cierto gasto de gobierno que resulta productivo y de gran valía para el crecimiento económico, pues permite elevar la rentabilidad de los agentes económicos privados mediante personal más capacitado y saludable, además de bienes de capital que les brinda ahorros en sus actividades diarias. No obstante, se piensa que el gasto de gobierno puede tener un efecto que tan sólo desplaza la provisión privada por la pública, generando en algunas ocasiones distorsiones en los mercados, o bien que el gasto público no tiene efectos en el crecimiento en la medida que los agentes económicos son sometidos a una mayor imposición de contribuciones en el futuro.

El gasto del gobierno y su efecto

en la inversión y el consumo privado

Como ya se mencionó, se tiene interés en dos tipos de efectos que puede generar el gasto público. El primero relacionado al gasto de capital que realiza el gobierno y que tiende a desplazar al gasto de capital del sector privado, asumiendo que ambos sectores pelean por los mismos recursos. El segundo que tiene que ver con el gasto que realiza el gobierno y que no aporta necesariamente mayor demanda en la economía, pues los agentes económicos, ante la expectativa de un incremento en sus impuestos futuros para financiar el gasto adicional, proceden a realizar ajustes en su consumo (TER).

Efecto sobre la inversión

Por tanto, una consideración en el análisis del gasto público es el relacionado con el efecto que tiene sobre la inversión privada; se pueden presentar tres efectos a saber: que resulte neutral, que tenga un efecto crowding-out o bien un efecto crowding-in.

La posición de neutralidad, derivada de los modelos neoclásicos, asume que cuando los agentes privados anticipan un incremento en la demanda generada por las 
expectativas de crecimiento del gasto de gobierno, los salarios se elevan anticipando el aumento de precios. La oferta agregada contrarresta cualquier efecto de expansión sobre el producto real (Erenburg y Wohar, 1995).

Tanto el efecto de crowding-out como el de crowding-in pueden aparecer, pero no hay elementos para pensar que se pueda tener un efecto neutral. Aschauer (1989b) expone que el efecto neto de un incremento del gasto de inversión pública probablemente aumente la inversión privada, todo dependerá de la política de gasto, pues el gasto público en ciertos rubros jugará un papel complementario a la inversión privada, permitiendo un mayor impacto en la producción (Aschauer, 1988). El autor encuentra que el multiplicador sobre la producción del gasto público en inversión es mayor que la unidad, mientras que el consumo público tiene un multiplicador menor a la unidad. Tridimas (1992) —en un trabajo que se enfoca al consumo privado más que a la inversión - indica que para capturar los efectos del gasto en el consumo privado es necesario desagregarlo. El gasto dedicado a los bienes públicos, como la administración pública, defensa, la ley y el orden — debido a que estos bienes no son del interés del mercado-, podrían generar un efecto de inclusión (crowding-in). En el gasto que se torna como sustituto directo del consumo privado, como la educación y la salud - debido que estos servicios pueden ser adquiridos en el mercado-, es posible pensar que el gasto público puede causar un crowding-out, pero esta idea sólo puede ser avalada si se considera que ambos sectores dedican su gasto a los mismos fines. Y, por último, es posible considerar que el gasto de capital genera efectos positivos en la economía en la medida que aporta mayor rentabilidad a la inversión privada.

\section{Efecto sobre el consumo}

El Teorema de la Equivalencia Ricardiana (TER) argumenta que un déficit público derivado de una reducción de los impuestos no expulsará inversión privada porque los consumidores aumentarán su ahorro, en lugar de incrementar el consumo corriente, en anticipación de las mayores obligaciones tributarias futuras.

El TER parte de la idea esbozada por David Ricardo y fuertemente retomada por Robert Barro (1974, 1996), en la cual se considera que se cuenta con dos medios de financiamiento del gasto público: ${ }^{4}$ los impuestos y la deuda. Sin embargo, bajo ciertas consideraciones no importa si el financiamiento del gasto se hace mediante

4 Para Barro (1996), con el planteamiento del TER, se avanza en la visión que resume los impactos de la PF por el lado del gasto y con ello se retoman las posibles distorsiones que se generan con los instrumentos de financiamiento del Estado. 
un incremento de impuestos o de deuda en el presente, pues en el futuro los agentes económicos racionales esperarán, si se elevó la deuda, un aumento en los impuestos. En este sentido, se puede inferir que en el largo plazo existe tan sólo un instrumento de financiamiento (impuestos).

La implicación de fondo del TER consiste en establecer que si el déficit presupuestal es financiado mediante la venta de bonos gubernamentales, ello no tendrá efectos en la demanda agregada de la economía porque las personas no aumentarán su consumo ante la expectativa de que en el futuro se eleven los impuestos; por tanto, la decisión de la venta de bonos es tan sólo una decisión de posponer el incremento en los impuestos.

Cuando se toma la decisión de disminuir los impuestos y se pone en marcha la emisión de bonos, en virtud de que el gasto de gobierno permanece constante, se supone que los agentes privados dedican parte de su ingreso disponible para adquirir los bonos. Ahora, ¿estos títulos han incrementado la riqueza neta de los poseedores? De acuerdo con Barro (1974), no existe un aumento en la riqueza neta porque el valor de adquisición de los bonos es igual al valor presente de los impuestos futuros. Por tanto, no se registrarán aumentos en el gasto de consumo y la estrategia fiscal no incide sobre la demanda agregada.

Considerar que una disminución de impuestos presente tendrá como consecuencia un incremento futuro dependerá de que la disminución de impuestos o el incremento del gasto, es decir una postura deficitaria, ${ }^{5}$ genere condiciones de crecimiento económico. En una situación en donde la tasa de crecimiento es mayor que la tasa de interés, no hay razón para pensar que los impuestos tendrán que elevarse como resultado del déficit actual. ${ }^{6}$

Arestis y Sawyer (2003), lo explican de la siguiente forma:

Asumiendo un déficit presupuestal (primario, es decir excluyendo el pago de intereses sobre la deuda) como proporción del PIB de "d", puede ser fácilmente mostrado que la deuda del gobierno para la proporción del PIB convergería en $\mathrm{b}=\mathrm{d} /(\mathrm{g}-\mathrm{r})$ donde "g" es la

5 Ireland (1994) comenta que una reducción en la tasa marginal del impuesto que conduce a un déficit presupuestal grande en el corto plazo podría ser cubierto en el largo plazo sin recurrir a un incremento subsiguiente de la tasa impositiva, esto ocurre porque una reducción presente de la tasa impositiva eleva la tasa de crecimiento económico y genera mayores ingresos tributarios aun con una tasa marginal del impuesto menor.

6 Domar (1944) expone un análisis teórico-empírico, en donde si la economía crece porcentualmente por encima de la tasa de interés que paga la deuda, la relación deuda-ingreso nacional además de permanecer estable en el tiempo puede disminuir a pesar de continuar con una política de contratación de deuda permanente. 
tasa de crecimiento del PIB y " $r$ " es la tasa de interés real sobre la deuda del gobierno. Si " $\mathrm{g}>\mathrm{r}$ ", entonces la deuda con relación al PIB se estabilizará aunque el monto de la deuda pendiente continuará aumentando (de acuerdo con el PIB). Pero, si g < r, entonces la deuda para la proporción del PIB no se estabilizaría y los intentos de dirigir un déficit presupuestario ininterrumpido llevarían a aumentar la deuda del gobierno. Debe, sin embargo, ser notado que si g $<\mathrm{r}$, entonces cualquier déficit llevará a aumentar la deuda con relación al PIB, aunque obviamente la acumulación será más lenta mientras más pequeño sea el déficit presupuestario. Por otro lado, si $\mathrm{g}>\mathrm{r}$, entonces cualquier tamaño de déficit presupuestario puede ser mantenido.

Siempre y cuando el déficit conlleve una estrategia de crecimiento, los agentes privados podrán tener la seguridad de que el incremento de su riqueza neta es real. Desde luego no debe olvidarse que bajo las condiciones de economía abierta, los efectos del déficit presupuestal pueden filtrarse al exterior sin arrojar los beneficios deseados.

Endogenidad del gasto de gobierno

Un tratamiento que no realizan normalmente los MCE consiste en la suposición de que el gasto de gobierno pudiera resultar endógeno, es decir, que en lugar de que el gasto de gobierno explicara el ingreso nacional, el ingreso explicara al gasto. Bleaney et al. (2001) menciona que uno de los problemas importantes de las evidencias empíricas es el concerniente a la exogenidad de las variables de los regresores de una ecuación de crecimiento, detalla que el rápido crecimiento puede inducir a mayores gastos públicos e incremento en las tributaciones o viceversa, o bien que se dé un efecto feed-back. El problema consiste en que si las variables fiscales no son estrictamente exógenas la evidencia empírica será engañosa.

De acuerdo con los modelos macroeconómicos de corte keynesiano, se ha considerado el gasto de gobierno como un instrumento exógeno de política que genera cambio en el ingreso nacional. Mientras que la denominada Ley de Wagner establece que la causalidad fluye de forma contraria, debido a que será el mayor crecimiento económico lo que propiciará los cambios en los niveles de gasto gubernamental.

La razón de profundizar en este planteamiento no consiste en llegar a una conclusión de si el crecimiento económico determina el aumento del gasto gubernamental, sino en todo caso la implicación que ello tiene: si el gasto público no juega un papel en el impacto al crecimiento de los países y sólo es causa, entonces las políticas económicas que utilizan el gasto de gobierno como instrumento de crecimiento no tienen sentido. 


\section{Ingresos tributarios}

Se sabe que todos los impuestos tienen un carácter distorsionador en la economía; salvo aquellos denominados de capitación, se dice que un impuesto distorsiona si interfiere en las decisiones privadas de los agentes económicos respecto al ahorro, la inversión y su predisposición al trabajo. No obstante, los problemas relacionados con dichas distorsiones pueden ser subsanados por las externalidades que se desprenden de la asignación de recursos a la población.

Como ya se mencionó, todos los impuestos distorsionan, pero algunos van dirigidos directamente a ciertas áreas que son estratégicas para el crecimiento. Si se asume que la inversión es un elemento detonador del aumento cualquier impuesto que conlleve una disminución en la misma irá en contra del crecimiento económico (Myles, 2000). Por tanto, aun cuando se estima que todos los impuestos distorsionan, no todos afectan en la misma proporción la generación de crecimiento y se hace necesario establecer una distinción entre los tipos de impuestos que conforman la estructura tributaria.

Existen coincidencias al respecto de que es preferible gravar con impuestos indirectos (consumo) que con impuestos al rendimiento del trabajo, mientras que la peor opción de gravamen sería sobre los rendimientos de los factores acumulables (King y Rebelo, 1990; Rebelo, 1991; Milesi-Ferreti y Roubini, 1998; y Doménech y García, 2001, entre otros).

Imposición de contribuciones

La imposición de contribuciones puede estudiarse desde dos ámbitos diferentes. Por un lado, como instrumento de PF que permite financiar el gasto que el gobierno realiza en la asignación de bienes y servicios; y, por otra parte, por los efectos que tiene en el crecimiento económico; este último ha sido objeto de estudios que intentan aportar evidencias acerca de la relación que guarda la imposición de contribuciones y el crecimiento de la economía. Myles (2000), bajo la teoría del crecimiento endógeno, comenta que el crecimiento económico está basado en el incremento de la productividad de la inversión de capital físico y humano, entre otros.

Por tanto, un impuesto que disminuya el rendimiento de la inversión puede tener efectos negativos en los niveles de crecimiento; el mecanismo de transferencia consiste en que "un incremento sobre la renta disminuye la tasa de rendimiento de las actividades de inversión del sector privado y conduce a un declive permanente en la tasa de acumulación del capital y en la tasa de crecimiento" (Rebelo, 1991). 
En este sentido, Easterly y Rebelo (1994) exponen que los impuestos sobre la inversión y el ingreso tienen un efecto perjudicial sobre el crecimiento. Por su parte, Engen y Skinner (1996), en un estudio que relaciona la tributación con el crecimiento, mencionan que si bien una tasa impositiva menor tiene un modesto efecto en el crecimiento económico, éste no garantiza que sea suficiente para que el recorte del impuesto se pague a sí mismo y, por tanto, se dará un deterioro en el nivel de vida. ${ }^{7}$

La reducción en los impuestos (the tax cut) puede ser vista como una medida que alienta el crecimiento, pero Gale y Orszag (2003) sostienen que debe tenerse cuidado, pues un recorte en los impuestos puede afectar al ingreso nacional propiciando la acumulación de capital humano, el ahorro, la inversión, a los empresarios, etcétera, pero la reducción de los ingresos incrementará el déficit y reducirá el ahorro nacional; por tanto, el efecto neto de la disminución de los impuestos será la suma de los dos momentos y el crecimiento dependerá de cuál de los dos tenga el mayor efecto.

Impuestos sobre el trabajo

Un impuesto sobre el trabajo puede tener dos efectos: el de ingreso y el de sustitución. Un incremento de la tasa de impuesto al trabajo disminuye la rentabilidad del mismo, y por tanto si los trabajadores quieren mantener su nivel de ingreso tendrán que optar por aumentar sus horas de trabajo. Sin embargo, asumiendo que el ocio es un bien normal, ahora los individuos sustituyen trabajo por ocio. Mientras que en el efecto sustitución se restringe la oferta de trabajo, en el efecto ingreso se incrementa; el resultado en términos absolutos puede ser neutral en la medida que se compensen ambos efectos (Macón, 2002, 87-88) o bien se podría concluir que el efecto completo de un impuesto sobre el trabajo es ambiguo (Zagler y Durnecker, 2003). Pero se tiene una situación en la que el impuesto sobre el trabajo puede ser desestabilizador y esto ocurre por la discrecionalidad de su manejo; si el gobierno confía en que los cambios en el impuesto pueden eliminar los desequilibrios presupuestales en el corto plazo, el desequilibrio se autovalidará por la percepción de los agentes económicos (SchmittGrohe y Uribe, 1997).

7 Zagler y Durnecker (2003) avanzan en el efecto de los impuestos sobre el crecimiento al considerar que si éstos tienen el objeto de financiar el gasto de gobierno y éste se traduce en un impacto positivo al crecimiento, entonces el efecto completo de los impuestos sobre el crecimiento debería ser la diferencia negativa de los impuestos causada por sus distorsiones menos el efecto positivo del gasto productivo del gobierno. 
Si la característica inherente de un impuesto sobre el trabajo es que disminuye los salarios netos, ello tendrá un efecto negativo en la acumulación de conocimientos, ya que la motivación por adquirir más y mejores conocimientos y habilidades está relacionada con la expectativa de ganar salarios futuros más elevados; Trostel (1993) y Zagler y Durnecker (2003) argumentan que los trabajadores aumentarán su escolaridad hasta el punto donde los beneficios marginales sean iguales a los costos marginales. ${ }^{8}$ Por tanto, un incremento en la tasa impositiva sobre el trabajo altera tanto los beneficios como los costos y por consecuencia el promedio de la calidad de la fuerza de trabajo y el crecimiento. ${ }^{9}$ Para autores como Feldstein y Vaillant (1994) un impuesto progresivo, del tipo de los impuestos al trabajo, implica que el costo para las empresas se eleva al contratar trabajadores con amplias capacidades en virtud de que los salarios se ajustan rápidamente a los cambios en el comportamiento de los impuestos, de esta manera las empresas contratarán menos trabajadores altamente calificados y más trabajadores menos calificados, teniendo también serias consecuencias para la acumulación de las habilidades. Independientemente de que los impuestos afecten o no los salarios netos, se tendrá un efecto negativo en la acumulación del factor trabajo, incidiendo con ello en el incremento del nivel de productividad y, por tanto, en el crecimiento económico.

Impuestos al capital

Un impuesto al capital reduce la tasa neta de rendimientos de la inversión en capital físico, por tanto incrementa los precios relativos del consumo futuro y consecuentemente alienta el consumo presente. Esta distorsión impositiva altera el comportamiento de los agentes económicos, desalentando la acumulación de capital físico, y por ello genera pérdidas en el crecimiento económico.

No obstante, una disminución en la tasa de rendimiento no implica necesariamente una sustitución al consumo presente; puede ser que esos recursos sean destinados a la formación de capital humano siempre y cuando éste ofrezca un mejor rendimiento. Un impuesto sobre los rendimientos de la inversión física puede tener influencia positiva sobre el capital humano; el impuesto reduce el rendimiento sobre la inversión

8 Donde los beneficios marginales se refieren a la expectativa futura de salarios más altos y los costos a las cuotas de colegiatura y al salario actual o pasado.

9 Bajo el enfoque de los MCE, se establece la formación de capital humano como una de las principales bujías para el crecimiento, por tanto cualquier impuesto que afecte la acumulación del mismo impide el crecimiento económico. 
física que hace al capital humano relativamente mejor inversión, por tanto la imposición sobre el capital físico puede alentar la sustitución por capital humano (Heckman, 1976). El efecto en el crecimiento al final dependerá de la especificación de la función de producción y particularmente de qué tipo de capital actúa como factor generador de crecimiento.

Impuestos al consumo

Los agentes económicos destinan sus ingresos ya sea para consumir o para ahorrar; las decisiones de qué parte se destina al consumo o al ahorro dependerán de la tasa de interés que se paga por posponer el consumo. Si un impuesto al consumo tiene la capacidad de modificar las decisiones entre la relación consumo-ahorro, entonces éste puede alterar los niveles de crecimiento, bajo el enfoque de los MCE, donde el ahorro podría jugar un papel muy importante.

Como se comentó, ante la imposibilidad de contar con un impuesto de suma fija o de "capitación" es necesario integrar una estructura fiscal que permita o propicie el crecimiento, para lo cual deberá ser lo menos distorsionante posible; en este sentido Doménech y García (2001) expresan, a partir de un estudio para los países de la Organización para la Cooperación y Desarrollo Económicos (OCDE) en el periodo 1960-1995, que los impuestos indirectos tienen un menor impacto para el crecimiento, y por tanto se postulan a favor de una disminución o intercambio de impuestos directos por impuestos indirectos, mencionando que la inequidad que se derive de este cambio en la estructura tributaria podría ser resuelta mediante programas de gasto, aun cuando es cuestionable si la PF sustentada en impuestos al consumo que resultan regresivos puede, mediante acciones de gasto, lograr la equidad en la economía.

\section{La disciplina fiscal y las fluctuaciones de corto plazo}

Cuando se asume la disciplina fiscal en los MCE, es decir que el gasto del gobierno debe ser igual al de los ingresos por concepto de contribuciones, se genera un contexto en el cual la capacidad de la PF para incidir en el crecimiento de corto plazo puede dejar de ser funcional e influir en el crecimiento de largo plazo, en virtud de que las fluctuaciones económicas que generan mayor volatilidad inhiben la consolidación del crecimiento en el mediano y largo plazos. De acuerdo con Ramey y Ramey (1994), no hay elementos para separar el crecimiento de las fluctuaciones económicas, ya que en países que tienen alta volatilidad se identifica que tienen menor crecimiento; 
por su parte Martner (1998) también asume que la volatilidad, medida como la desviación estándar de la tasa de crecimiento anual, juega un papel importante entre los factores de crecimiento denotando que para los países de América Latina las amplias fluctuaciones conllevaron a bajas tasas de crecimiento en los ochenta y parte de los noventa del siglo pasado. En este sentido, diferentes economistas han expresado la necesidad por el uso de políticas contra-cíclicas que disminuyan la inestabilidad del sistema de producción capitalista (Ramírez, 2006).

La inestabilidad en la tasa de crecimiento de ciertos países se debe al tipo de política económica que llevan a cabo y que tienen que ver con el planteamiento de finanzas públicas sanas, que configuran una postura pro-cíclica en sus variables fiscales de gasto-ingreso.

La relación que tiene la PF respecto al ciclo económico puede adoptar por lo menos tres padrones de conducta: a-cíclica, pro-cíclica o contra-cíclica; en el primero de los casos se asume que la PF no tiene relación con el ciclo económico o bien que de existir la relación ésta es muy débil; cuando se menciona que una PF es pro-cíclica se asume que sigue los movimientos del ciclo económico, mientras que cuando es contra-cíclica se argumenta que se mueve de forma contraria al ciclo. El comportamiento del presupuesto fiscal puede estar determinado por dos causas diferentes: el reflejo del entorno económico o bien el resultado de decisiones políticas discrecionales. ${ }^{10}$ Ante una postura de PF discrecional que se mantiene a-cíclica el entorno económico conducirá al presupuesto fiscal a un comportamiento contra-cíclico, es decir, se incrementará el déficit en recesión y disminuirá conforme se recupere la economía. Cuando se elimina el componente discrecional del ciclo económico se asume que la PF tendería de manera automática a ser contra-cíclica. ${ }^{11}$

No obstante, una PF que persigue el equilibrio presupuestal independientemente de la fase del ciclo es probable que arroje una postura fiscal pro-cíclica, cuando es aplicada en un contexto de estancamiento o bajo crecimiento económico, pues, de acuerdo con el ciclo, los ingresos públicos serán bajos al igual que el gasto gubernamental, conduciendo probablemente a contextos de inestabilidad y volatilidad que no garantizan una trayectoria de crecimiento de largo plazo.

10 "Sistemáticamente uno puede pensar que cambios en las variables fiscales son la suma de dos componentes: El primero refleja el ajuste automático de las variables fiscales al ambiente económico subyacente; mientras que el segundo son los cambios discrecionales aplicados por los responsables de la PF" (Gavin y Perotti, 1997).

11 Vía el uso de estabilizadores automáticos. 


\section{La coyuntura del déficit fiscal}

Bajo el paradigma neoclásico de pleno empleo se asume que un incremento en el gasto de gobierno deficitario puede tener efectos negativos sobre el crecimiento económico, no obstante diversos economistas han expuesto - sobre una base del pensamiento keynesiano-kaleckiano y sobre los desarrollos de Harrod y Domar-que en las economías capitalistas se asume la existencia de capacidad ociosa o exceso de capacidad (Moudud, 1998), además de que la dinámica de largo plazo es perfectamente consistente con desempleo (Shaikh, 1989).

Una explicación para relajar el supuesto de pleno empleo puede ser que los niveles de producción no están determinados por las capacidades productivas, sino más bien por la posibilidad de venta o demanda efectiva. ${ }^{12}$ En un sistema capitalista es frecuente encontrar recursos ociosos. ${ }^{13}$ Kalecki (1973) mencionaba que el grado de utilización del equipo es muy pequeño durante la depresión, dando como contrapartida el desempleo de la fuerza de trabajo, el argumento para no producir con equipo no utilizado radica en el poco beneficio que se obtiene, en virtud que los precios de venta están por debajo de los costos de producción, la reducción de los salarios podría compensar la relación costo-precios, pero la generalización de la reducción salarial puede operar de manera contraria al reducir la posibilidad de la realización de los bienes producidos, dada la disminución de la demanda efectiva. ${ }^{14}$

Cuando la economía opera con capacidad ociosa, la solución debería ser el incremento de la demanda efectiva, mediante incentivos a la inversión (como los que se derivan de cierto manejo tributario y menor tasa de interés) o el gasto de gobierno; la primera opción parece ser mejor para evitar la participación del gasto público, no obstante es necesario tener presente el problema de Domar, que deviene de una situación en la cual el incremento de la demanda originado por un crecimiento en la inversión puede ser insuficiente para cubrir la nueva capacidad productiva que fue generada por el aumento de la inversión. Wray (2007) comenta que el problema de Domar refuta

12 Keynes $(2003 ; 60)$ argumenta que una explicación de la paradoja de la pobreza en medio de la abundancia es la existencia de una demanda efectiva insuficiente que a menudo hará que el aumento de la ocupación se detenga antes de que se haya alcanzado la ocupación plena.

13 En algunos casos el no trabajar a $100 \%$ de la capacidad instalada responde a estrategias de mercado de las empresas.

14 Kalecki (1973, 58-59) indicaba que la disminución salarial incrementa los beneficios de los capitalistas, por tanto si éstos aumentan su consumo en la misma proporción que los bienes no consumidos por los asalariados se conseguiría un equilibrio en un nivel más elevado, pero generalmente el consumo de los capitalistas cambia muy poco en el ciclo económico. 
la Ley de Say, en virtud de que un estímulo a la oferta no sería acompañado de una oferta suficiente, por tanto estimular la inversión en un escenario donde la demanda resulta ser insuficiente no es la mejor opción; en este caso, el gasto gubernamental deficitario debería absorber la capacidad creada por la inversión privada. ${ }^{15}$

\section{La dinámica de las ganancias}

En un sistema de producción de corte capitalista se sabe que la conducta de los agentes económicos está determinada o bien depende de la generación de ganancias. Las ganancias se constituyen como una fuente importante de acumulación de largo plazo, por tanto cualquier variable que afecte el comportamiento de las ganancias también tendrá efectos sobre el crecimiento.

Al seguir con la formulación planteada por Kalecki (1984) se tiene que las ganancias en este sistema van a depender de la inversión de los agentes privados, del gasto de gobierno más allá de los recursos que extrae de los agentes económicos y del comercio exterior neto, todo ello menos el ahorro del sector privado. Lo anterior se podría expresar:

$$
\text { Ganancias }=\mathrm{I}+(\mathrm{G}-\mathrm{T})+(\mathrm{X}-\mathrm{M})-\mathrm{S}
$$

Esta identidad contable resulta de suyo muy importante en la medida que se tienen los tres sectores de la economía: el privado, el público y el externo. Al reescribir la ecuación:

$$
(\mathrm{S}-\mathrm{I})=(\mathrm{G}-\mathrm{T})+(\mathrm{X}-\mathrm{M})
$$

Se puede decir que las ganancias están determinadas por la inversión, el gasto deficitario del sector público, las exportaciones netas, el consumo capitalista y de forma negativa el ahorro de los trabajadores. Siempre que se realiza el supuesto de que los trabajadores gastan lo que ganan, se tiene que la economía puede crecer con

15 La distinción del gasto público bajo un contexto de exceso de capacidad puede perder su contundencia. En condiciones normales se asume como una regla de oro, en materia de PF, que los gastos corrientes deban de ser cubiertos con ingresos corrientes; por tanto, el gasto en consumo no debería de ser deficitario. Sin embargo, cuando las condiciones no son normales y se tiene capacidad ociosa, el gasto deficitario, aun cuando sea en consumo, debería tener efectos sobre la tasa de ganancias incrementando la capacidad utilizada y a su vez la inversión (Moudud, 1998). 
un superávit comercial, un déficit fiscal o un déficit del sector público o bien una combinación de los tres.

Para Kalecki el gasto gubernamental es importante para la determinación de las ganancias agregadas; él asume que la inversión y el déficit de gobierno constituyen el factor causal que inicia el proceso de generación de ganancias (Parguez, 1991). En una situación de exceso de capacidad, la elección sería el gasto de gobierno deficitario; aquel que no genera presiones sobre el ingreso de los agentes económicos, ${ }^{16}$ que puede incrementar la demanda efectiva, el déficit presupuestal viene a dinamizar la economía al incidir sobre las ganancias.

\section{La disciplina fiscal y el papel de los impuestos}

En aquellas economías que han adoptado la disciplina fiscal como regla de política se podría pensar que de manera implícita han asumido que los impuestos son la única forma de financiar el gasto del gobierno. Lo anterior tiene por lo menos las siguientes implicaciones: se impone al Estado una restricción presupuestal tal como si fuera un agente privado más en la economía, ${ }^{17}$ la capacidad de la PF para influir en el ciclo económico y aminorar sus fluctuaciones son limitadas; y, de manera adicional, cuando los impuestos funcionan bajo la lógica de ser tan sólo medios de financiamiento, dejan de lado su carácter de mecanismos con los cuales se logra la equidad del ingreso y la riqueza en la sociedad.

El escenario en el cual se consolida una regla de política como la mencionada tiene que ver con la pérdida del manejo soberano de la moneda por parte del Estado, ${ }^{18}$ perdiendo también con ello la capacidad de establecer políticas económicas soberanas. Un país que tiene el manejo soberano de su moneda emplearía los impuestos de

16 Aquí Kalecki hace la precisión de que no es necesariamente con el déficit presupuestal como se genera una demanda efectiva adecuada; si el gravamen se establece sobre los ahorros de los beneficios puede generar situaciones de pleno empleo, pero si se establece sobre los beneficios es probable que no se tenga una variación importante en la inversión ni en el consumo.

17 A decir de Mosler y Forstater (2004), la distinción clave entre el gobierno y los ciudadanos consiste en que el primero es el emisor de la moneda y los segundos son los usuarios de la misma. Operacionalmente el gasto público consiste en abonar en la cuenta de un banco miembro del banco central o pagando con efectivo. Por tanto, a diferencia de los usuarios de la moneda, y en dirección opuesta a la concepción popular, el emisor de la moneda no restringe su gasto a sus ingresos.

18 El dinero del Estado pierde su soberanía cuando se debe garantizar su convertibilidad a un metal precioso o a alguna moneda externa, tal como lo expresa Randall Wray en diferentes publicaciones. 
una forma diferente a la convencional que tan sólo les asigna un papel de tesorería. El punto fino de esta disyuntiva radica en que, sin importar que muchos países pudieran tener la capacidad de operar una moneda soberana, ellos mismos han declinado esa posibilidad al establecer la independencia de su banco central. ${ }^{19}$

La restricción a la que se ha autosometido el Estado es consistente con la teoría neoclásica que pugna por su salida de las actividades económicas, abriendo paso a los particulares en la realización de obras de infraestructura que son necesarias y que no le es posible realizar al Estado por su restricción presupuestal, pero en las cuales pueden estar ampliamente interesados los particulares por su alta rentabilidad.

Cuando un país retoma de nueva cuenta el manejo soberano de su moneda, el papel que deberían de tener los impuestos puede ser suscrito a tres aspectos fundamentales: la creación de demanda por la moneda del Estado, la regulación del gasto privado y la redistribución del ingreso y la riqueza.

Impuestos como generadores

de demanda por el dinero del Estado

Los impuestos tienen la función de crear demanda por el dinero que el Estado ha emitido; cuando éste es capaz de imponer contribuciones a los ciudadanos y determinar con qué medio de pago se han de liquidar dichas obligaciones, se estará logrando la aceptación generalizada de "aquello" que es recibido en las oficinas de la hacienda pública. Los impuestos, además de lograr la aceptación generalizada por parte de la sociedad que es contribuyente, también tienen el objetivo de mantener su valor. En las economías modernas donde el dinero tiene por un lado un valor nominal y por otro un valor intrínseco que no coinciden entre sí y que no necesariamente tendrían que hacerlo, los impuestos impiden que el valor nominal del dinero se pierda. ${ }^{20}$

Desde esta concepción, el Estado no necesita el dinero de los particulares para gastar, son los particulares quienes necesitan el dinero del Estado para cumplir con

19 La razón de tener un banco central autónomo se suscribe a la visión ortodoxa que asume a la emisión de dinero como una de las causas de los procesos inflacionarios, pues se piensa que si el gobierno puede tener injerencia en el banco central es posible que use ese poder para sus propios objetivos, mismos que en ocasiones tienen poco que ver con el desempeño de la economía y tienen tintes de carácter político. La autonomía de la banca central descarta la soberanía de la moneda y obliga al gobierno a restringir su gasto a los ingresos que pueda extraer de la sociedad vía recaudación de impuestos.

20 De acuerdo con Wray (2006), los impuestos son necesarios para darle valor a la moneda, ya que si el dinero de emisión primaria fuera de fácil obtención, podría perder su valor porque no se necesita trabajar arduamente para conseguirlo. 
sus obligaciones fiscales (Wray, 2002). La relación lógica entre gasto-ingreso empieza cuando el gobierno crea y gasta dinero nuevo para que luego la población pague sus impuestos; si no cómo se podría explicar que la población cumpliera con sus obligaciones fiscales usando el dinero del Estado antes de que éste lo hubiera creado y puesto a la disposición de ellos (Bell, 1998). Como se puede apreciar bajo el contexto de soberanía monetaria, el gobierno no está restricto a la recaudación de ingresos tributarios para poder incrementar su gasto.

Impuestos como reguladores

del gasto privado

El manejo de un sistema tributario sirve para reducir las disponibilidades líquidas de los contribuyentes, es decir, la tasa impositiva se debe de mover de acuerdo con el grado de liquidez que estime conveniente el gobierno. Si se está ante la presencia de un Estado soberano capaz de crear su propio dinero, se tiene entonces que las decisiones en materia de contribuciones sólo deberán tomarse de acuerdo con los efectos económicos que desee el Estado en sus variables objetivo, y no por su necesidad de recursos para financiar el déficit presupuestal. Los impuestos deben jugar el papel de regular de manera indirecta el gasto de los agentes privados y permitir mayor o menor gasto de acuerdo con el ciclo económico y bajo esta lógica el nivel de recaudación fiscal debería de ser pro-cíclica, teniendo menor cuantía en la fase baja del ciclo y una mayor cuantía en la parte alta, sin que ello pueda ser considerado como una situación anormal.

Impuestos como redistribuidores

del ingreso y la riqueza

En un sistema de producción capitalista la concentración del ingreso y la riqueza son elementos inherentes al propio sistema. Por tanto, la idea de que el crecimiento económico puede propiciar una mayor y mejor redistribución del ingreso y la riqueza no es cierta por sí misma. La redistribución se podría conseguir si en términos generales la estructura tributaria resultara ser más progresiva que regresiva; es decir, que aquellas personas que tuvieran mayores ingresos contribuyeran con mayores impuestos. Lo anterior no sólo por un carácter altruista o equitativo, sino también con el objetivo de elevar el gasto en la economía y generar niveles de mayor demanda y por tanto oportunidades de inversión.

El centro de atención radica en los efectos que tienen los impuestos en los objetivos económicos; en este sentido la distribución del ingreso juega un papel importante 
al mejorar las condiciones del nivel de vida de las personas de menores ingresos garantizando con ello que su demanda no se vea mermada, ${ }^{21} \mathrm{y}$ por otra parte se garantiza que el gobierno introduzca, vía gasto, recursos adicionales a la economía, es decir, agrega gasto que no iba a ser realizado por las personas sujetas al impuesto.

$\mathrm{Si}$ se ha extendido la idea de que los impuestos son un mecanismo de financiamiento del Estado, es debido a la pérdida de la soberanía del dinero en economías donde se ha optado por la independencia del banco central. En este sentido se ha privilegiado el control de precios independientemente del perjuicio en las variables reales de la economía, lo que trae consecuencias negativas sobre la estabilidad macroeconómica de corto plazo e incidiendo en el mismo sentido sobre el crecimiento de largo plazo.

\section{Conclusiones}

En el marco de los MCE se piensa que la PF tiene una participación relevante, pero la misma se acota tan sólo a la buena distribución de los gastos en productivos e improductivos, así como en la correcta conformación de una estructura tributaria que distorsione la economía lo menos que sea posible.

En materia de gasto, los MCE tienen el elemento favorable de distinguir entre gastos que favorecen el crecimiento de largo plazo y aquellos que pueden resultar neutrales o no, dependiendo de si su financiamiento se realiza con ingresos tributarios o si éstos son financiados con deuda. No obstante, soslayar la discusión de tener un gasto productivo deficitario conlleva a una limitante en el planteamiento, sobre todo en economías en desarrollo.

La limitante del gasto de gobierno se da por el lado de su financiamiento al asumir que los impuestos son la fuente que menor afectación tiene para el crecimiento, a diferencia de la deuda y de la emisión de dinero que tienen efectos sobre la tasa de interés y la inflación que atentan contra la acumulación de capital. Es decir, los impuestos se toman como el menor de los males, no obstante se hace necesario también eliminar las distorsiones para el crecimiento que puedan causar. Para ello se antepone que los impuestos deben de observar eficiencia económica o, lo que es lo mismo, ge-

21 Como bien dice Wray (2004), cualquier medida de recortes tributarios debe ir encaminada a las personas de menores ingresos para asegurar que se tendrán menores efectos multiplicadores sobre el empleo, tanto en términos de la demanda como en términos de la oferta; en el primer caso estimulando el consumo y en el segundo alentando a los empleadores a contratar y a los trabajadores a trabajar. 
nerar ingresos que afecten lo menos posible los factores de acumulación. Tal acción conlleva a construir estructuras tributarias sustentadas en impuestos indirectos, sin tomar en cuenta que tal determinación puede provocar una mayor inequidad que atente contra la tasa de crecimiento en el corto y largo plazos; aun cuando se argumente que el gasto social puede atenuar la desigualdad, esto podría ser impreciso bajo dos consideraciones: gasto social no focalizado o bien no progresivo y el hecho de que la distribución para ser efectiva tendría que partir de la generación del ingreso que es donde se genera su concentración.

La relación que se hace entre corto y largo plazos trata de establecer un vínculo entre las acciones que se realizan día a día y los resultados obtenidos, bajo la visión de que el largo plazo es la suma de los cortos plazos, por lo que se hace necesario estabilizar las fluctuaciones de la economía para evitar sus efectos negativos sobre la trayectoria de la tasa de crecimiento.

La disciplina fiscal que es asumida por algunos como necesaria para generar condiciones de crecimiento de largo plazo es contradictoria en un contexto de déficit comercial, pues atenta contra la generación de ganancias y, a su vez, sobre la acumulación de capital y la tasa de crecimiento.

Las economías están sujetas a la disciplina fiscal cuando han declinado su soberanía monetaria, ante el temor de los procesos inflacionarios. Bajo esta condición los impuestos asumen como su principal función el financiamiento del gasto. Esta consideración impide que los impuestos jueguen los papeles descritos con anterioridad inhibiendo con ello que la PF pueda tener una postura contracíclica.

El déficit fiscal en momentos de recesión económica es de vital importancia para configurar condiciones endógenas de crecimiento que ayuden a superar la depresión. El déficit presupuestal se puede constituir como el estado normal de cualquier economía que busque objetivos de pleno empleo, crecimiento económico y nivel de precios estable. Es mediante el déficit fiscal sobre el que se configura la capacidad de una PF para adoptar una política contra-cíclica que tanta falta hace para evitar la volatilidad que presenta el crecimiento económico. No obstante, el déficit fiscal ha quedado fuera del uso de las economías modernas, siendo uno de los elementos principales en la discordia de las interpretaciones económicas que queda de manifiesto en el planteamiento y desarrollo de los MCE.

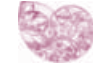




\section{Bibliografía}

Alesina, Alberto y Roberto Perotti, "Fiscal Adjustments in OECD Countries: Composition and Macroeconomic Effects", Working Paper, núm. 5730, Massachusetts, NBER, 1996.

Arestis Philip, y Malcom Sawyer, "Reinventing Fiscal Policy”, Working Paper, núm. 381, The Levy Economics Institute of Bard College, Nueva York, mayo de 2003.

Aschauer David, "Is Government Spending Stimulative?", Staff Memoranda, Chicago, Federal Reserve Bank of Chicago, 1988.

"Does Public Capital Crowd

Out Private Capital?", Journal of Monetary Economics, 24, Norte de Holanda, Elsevier, 1989b, pp. 171-188.

, "Is Public Expenditure Productive?", Journal of Monetary Economics, 23, Norte de Holanda, Elsevier, 1989a, pp. 177-200.

Barro, Robert y Xavier Sala-I-Martin "Public Finance in Models of Economic Growth", The Review of Economic Studies, vol. 59, núm. 4, Londres, London School of Economics and Political Science, octubre de 1992, pp. 645-662.

Barro, Robert, "Are Government Bonds Net Wealth?", The Journal of Political Economy, vol. 82, núm. 6, Chicago, The University of Chicago, nov-dic. 1974, pp. 1095-1117.

, "Economic Growth in Cross Section of Countries", The Quarterly Journal of economics, vol. CVI, núm. 425, Massachusetts, MIT, mayo de 1991, pp. 407.443.

, "Government Spending in a Simple Model of endogenous Growth", The Journal of Politic Economy, vol. 98, núm. 5, Chicago, The University of Chicago, octubre 1990, pp. 103-126.

"Reflections on Ricardian Equivalence", Working Paper, núm. 5502, Massachusetts, NBER, marzo de 1996.

Bell, Stephanie, "Can Taxes and Bonds Finance Government Spending?", Working Paper, núm. 244, Nueva York, The Levy Economics Institute of Bard College, julio de 1998.

Bleaney, Michael et al., "Testing the Endogenous Growth Model: Public Expenditure, Taxation and Growth over the Long-Run", Canadian Journal of Economic, vol. 34, núm. 1, Montreal, Canadian Economics Association, febrero de 2001, pp. 36-57.

Camara Neto, Alcino. y Vernengo Matías, "Fiscal Policy and the Washington Consensus: A Post Keynesian Perspective", Working Paper, núm. 9, Utah, Universidad of Utah, Department of Economics, 2004.

Devajaran, Shantayanan, et al., "The composition of public expenditure and economic growth", Journal of Monetary Economics, vol. 37, núm. 2, Norte de Holanda, Elsevier, 1996, pp. 313-344.

Domar, Evsey, "The Burden of the Debt and the National Income", The American Economic Review, vol. 34, núm. 4, Nashville, American Economic Association, diciembre de 1944, pp. 798-827.

Doménech, Rafael y José García, "Estructura fiscal y crecimiento económico en la OCDE”, Investigaciones Económicas, vol. XXV (3), Madrid, Fundación SEPI, septiembre de 2001, pp. 441-472.

Easterly, William y Sergio Rebelo, "Fiscal Policy and Economic Growth: An Empirical Investigation", Working Paper, núm. 4499, Massachusetts, NBER, octubre de 1994.

Engen Eric y Jonathan Skinner, "Fiscal Policy and Economic Growth", Working Paper, núm. 4223, Massachusetts, NBER, diciembre de 1992 .

, "Taxation and Economic Growth", National Tax Journal, vol. 49, núm. 4, Ohio, National Tax Association, diciembre de 1996, pp. 617-642.

Erenburg, S. J. y Wohar Mark, "Public and Private Investment: Are There Causal Linkages?", Journal of Macroeconomics, vol. 17, núm. 1, Louisiana, Lousiana State University Press, invierno de 1995, pp. 1-30.

Feldstein, Martin y Marian Vaillant, "Can State Taxes Redistribute Income?", Working Paper, núm. 4785, Massachusetts, NBER, septiembre de 1994.

Gale, William y Peter Orszag, "Fiscal Policy and Economic Growth: A Simple Framework", Tax Analysts, núm. 3, Tax Notes, http:// www.urban.org/url.cfm?ID=1000450, febrero de 2003, pp. 759-764.

Gavin Michael y Perotti Roberto, "Fiscal Policy in Latin America", Published in NBER Microeconomics Annual 1997, Massachusetts, NBER 1997. 
Gemmell, Norman, "Fiscal Policy in a Growth Framework", $D P$, núm. 48, Helsinki, UNU/ Wider, World Institute for Development Economics Research, septiembre de 2001.

Heckman, James, "A Life-Cycle Model of Earnings, Learning, and Consumption", Journal of Political Economy, núm. 84, Chicago, University of Chicago Press, agosto de 1976, pp. 11-44.

Ireland, Peter, "Supply-side Economics and Endogenous Growth", Journal of Monetary Economics, 33, Norte de Holanda, Elsevier, 1994, pp. 559-571.

Kalecki, Michael, "Estudio Sobre la Teoría de los Ciclos Económicos", 2ª ed., España, Ariel, 1973.

, Teoría de la dinámica económica, ensayos sobre los movimientos cíclicos y a largo plazo de la economía capitalista, , $4^{\mathrm{a}}$. reimpresión, México, FCE, 1984.

Keynes J., Teoría General de la Ocupación el Interés y el Dinero, $4^{\mathrm{a}}$. edición, México, FCE, 2003.

King, Robert y Sergio Rebelo, "Public Policy and Economic Growth: Developing Neoclassical Implications", The Journal of Political Economy, vol. 98, núm. 5, Chicago, The University of Chicago, 1990, pp. 126-150.

Lerner A., "Money as a Creature of the State", The American Economic Review, vol. 37, núm 2, Nashville, American Economic Association, mayo de 1947, pp. 312-17.

Macón, Jorge, Economía del Sector Público", Bogotá, McGraw-Hill, 2002.

Martner, Ricardo, "Política fiscal, ciclo y crecimiento" Revista de la CEPAL, núm. 64, Santiago de Chile, CEPAL, abril de 1998 , pp. 73-90.

Milesi-Ferretti, Gian y Nouriel Roubini, "Growth effectsof Income and Consumption Taxes" Journal of Money, Credit and Banking, vol. 30, núm. 4, Ohio, Ohio State University Press, noviembre de 1998, pp.721-744.

Mosler, Warrent y Mathew Forstater, "The Natural Rate of Interest is Zero", Working Paper, núm. 37, Kansas City, CFEPS, University of Missouri, diciembre de 2004.

Moudud, James, "Government Spending and Growth Cycles: Fiscal Policy in a Dynamic Context", Working Paper, núm. 260, Nueva York, The Levy Economics Institute of Bard College, diciembre de 1998.

Myles, Gareth, "Taxation and Economic Growth", Fiscal Studies, vol. 21, núm. 1,
Londres, Institute for Fiscal Studies, 2000, pp. 141-168.

Parguez, Alain "Keynesianismo y Austeridad", Investigación Económica, 198, México octubre-diciembre de 1991.

Ramey, Garey y Valerie Ramey, "Cross-Country Evidence on the Link Between Volatility and Growth", Working Paper, núm. 4959, Massachusetts, NBER, diciembre de 1994.

Ramírez, Eduardo, "Por una Política Fiscal Contra-cíclica". Problemas de Desarrollo, vol. 137, núm. 147, México, IIE-UNAM, octubre-diciembre de 2006, pp. 81-170.

Ramírez, Eduardo. "La función de los impuestos en una economía moderna con manejo de moneda soberana", Ponencia: XII Foro de Investigación: Congreso Internacional de Contaduría, Administración e Informática, FCA-UNAM, México, octubre de 2007.

Rebelo, Sergio, "Long Run Policy Analysis and Long Run Growth", The Journal of Political Economy, vol. 99, núm. 3, Chicago, The University of Chicago, 1991, pp. 500-521.

Romer, Paul, "Endogenous Technological Change", The Journal of Political Economy, vol. 98, núm. 5, Chicago, University of Chicago Press, 1990, pp. 71-102.

Schmitt-Grohe, Stephanie y Martin Uribe, "Balanced-Budget Rule, Distortionary Taxes, and Aggregate Instability", The Journal of Political Economy, vol. 105, núm. 5, Chicago, University of Chicago Press, octubre de 1997, pp. 976-1000.

Shaikh, Anwar, "A Dynamic to the Theory of effective Demand", Working Paper, núm. 19, Nueva York, The Levy Economics Institute of Bard College, marzo de 1989.

Tridimas, George, "A note on the effects of Government Expenditures on Private Consumption", Public Finance/Finances Publiques, vol. 47(1), Frankfurt, Foundation Journal Public Finances, 1992, pp.153-161.

Trostel, Philip, "The Effect of Taxation on Human Capital", The Journal of Political Economy, vol. 101, núm. 2, Chicago, University of Chicago Press. abril de 1993, pp. 327-350.

Wray, Randall, "Friedmanian Approach to Restoring Growth", Working Paper, núm. 22, Kansas City, CFEPS, University of Missouri-Kansas City, septiembre de 2002.

, "Understanding Policy in a Floating Rate Regime", Working Paper, núm. 51, Kansas City, CFEPS, University of Missouri-Kansas City, enero de 2006. 
"Demand Cosntraints and Big Goverment", Working Paper, núm. 488, Nueva York, The Levy Economics Institute of Bard College, enero de 2007.

"Money and Taxes: The Char-

talist", Working Paper, núm. 222, Nueva York, The Levy Economics Institute of Bard College, enero de 1998.
"Those "D" Words: Deficits, Debt, Deflation, And Depreciation", Policy Note, núm. 2, Nueva York, The Levy Economics Institute of Bard College, 2004.

Zagler, Martin. y Georg Dürnecker, "Fiscal Policy and Economic Growth", Journal of Economic Survey, vol. 17, núm. 3, Oxford, Blackwell Publishers, 2003, pp. 397-418.

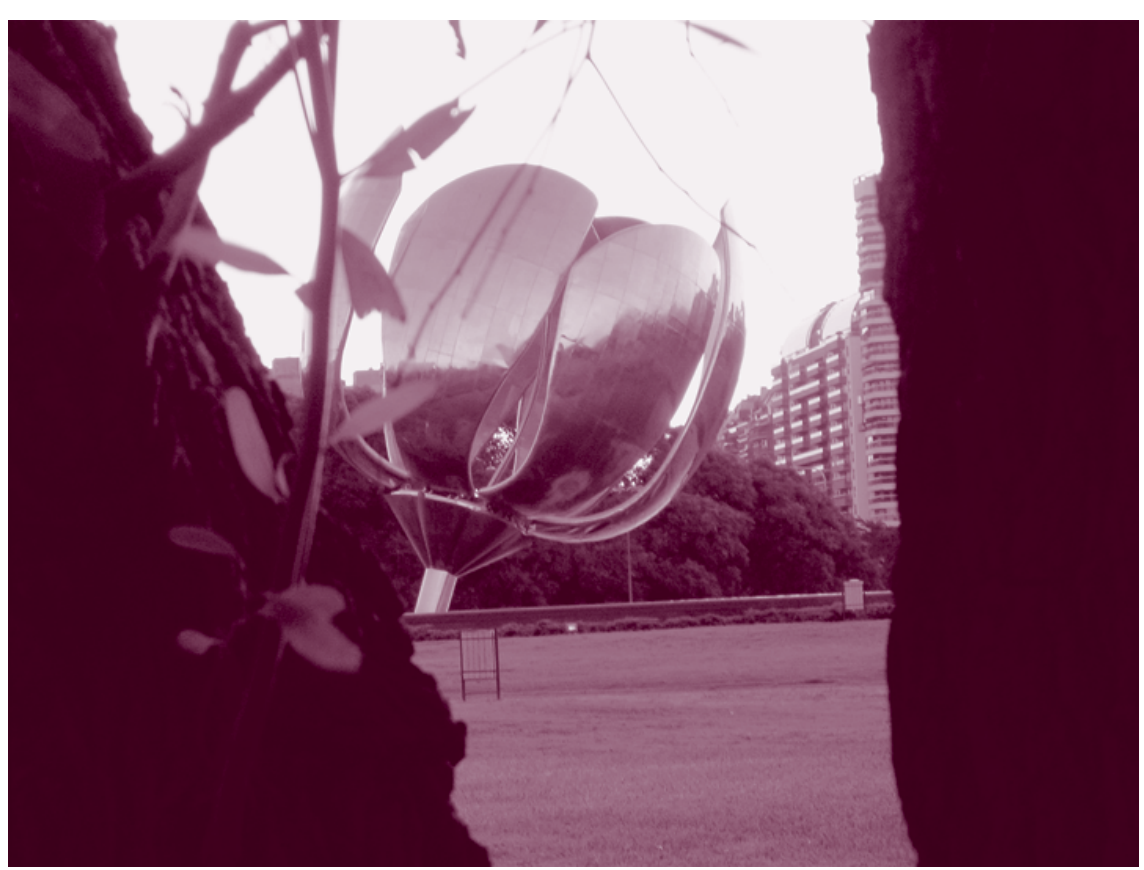

La flor III. Buenos Aires, verano 2005-2006. PAI 